\title{
Cetuximab improves AZD6244 antitumor activity in colorectal cancer HT29 cells in vitro and in nude mice by attenuating HER3/Akt pathway activation
}

\author{
QIN ZHANG ${ }^{*}$, HE XIAO* ${ }^{*}$ FENG JIN, MENGXIA LI, JIA LUO and GE WANG \\ Cancer Center, Research Institute of Surgery, Daping Hospital, The Third Military Medical University, \\ Chongqing 400042, P.R. China
}

Received September 11, 2017; Accepted March 15, 2018

DOI: $10.3892 / \mathrm{ol} .2018 .8674$

\begin{abstract}
The present study investigated the molecular mechanism by which the epidermal growth factor receptor (EGFR) inhibitor cetuximab enhances the antitumor activity of the mitogen-activated protein kinase kinase (MEK) inhibitor AZD6244 in colorectal cancer HT29 cells. HT29 cells were treated with AZD6244 plus cetuximab and then subjected to the following assays: Cell Counting kit-8, BrdU-incorporation, flow cytometric cell cycle distribution and apoptosis analysis, western blot analysis, and nude mouse xenografts. The combination of AZD6244 and cetuximab significantly reduced HT29 cell viability and proliferation compared with AZD6244 alone. The combination treatment reduced the $\mathrm{IC}_{50}$ value from $108.12 \pm 10.05$ to $28.45 \pm 1.92 \mathrm{nM}$. AZD6244 and cetuximab also induced cell cycle arrest at G1 phase and reduced S phase (88.53\% vs. $93.39 \%$, $\mathrm{P}=0.080 ; 8.73 \%$ vs. $4.24 \%, \mathrm{P}=0.082$, respectively). Combination of AZD6244 with cetuximab significantly induced tumor cells apoptosis (14.61\% vs. $8.99 \%, \mathrm{P}=0.046)$. Inhibition of EGFR activity using cetuximab partially abrogated the feedback-activation of phosphorylated receptor tyrosine-protein kinase erB-3 (p-HER3) and p-AKT serine/threonine kinase (AKT), as well as prevented reactivation of $\mathrm{p}$-extracellular regulated kinase (ERK) conferred by AZD6244 treatment. Combination of AZD6244 and cetuximab also inhibited HT29 cell xenograft growth in nude mice and suppressed HER3 and p-AKT levels in xenografts. The EGFR inhibitor cetuximab enhanced the antitumor activity of the MEK inhibitor AZD6244 in colorectal cells in vitro and in vivo. Co-inhibition of MEK and EGFR may be a promising treatment strategy in colorectal cancers.
\end{abstract}

Correspondence to: Dr Ge Wang, Cancer Center, Research Institute of Surgery, Daping Hospital, The Third Military Medical University, 10 Changjiang Zhilu, Chongqing 400042, P.R. China E-mail: wangge70@hotmail.com

*Contributed equally

Key words: cetuximab, AZD6244, colorectal cancer, feedback activation

\section{Introduction}

Colorectal cancer (CRC) is a significant health problem worldwide for men and women, and accounted for an estimated 1.4 million new cases and 693,900 cancer-associated mortalities globally in 2012, the global burden of the CRC is expected to increase by $60 \%$ to $>2.2$ million new cases and 1.1 million mortalities by $2030(1,2)$. Unhealthy diets, obesity and tobacco smoking are major risk factors for the incidence of CRC, particularly in several Asian and Eastern European countries (2). Despite the implementation of active cancer screening for early detection, prevention and advanced treatment strategies in the last decade, $\mathrm{CRC}$-associated morbidity remains a clinical burden $(1,3,4)$. Thus, identifying effective treatment regimens may aid in controlling or treating the advanced stages of the disease, and improve the 5-year overall survival rate of patients.

CRC, like most other human cancer types, is defined by an abnormal growth of cells that have the ability to invade or spread to other parts of the body (5). Molecularly, gene expression-based subtyping of CRC has identified four subtypes, named consensus molecular subtypes (CMSs) that are specifically associated with different characteristic mutations. For instance, mutations of proto-oncogene B-Raf (BRAF) have been frequently observed in the CMS1 subtype and KRAS proto-oncogene GTPase (KRAS) mutations are overrepresented in the CMS3 subtype (6). Knowledge of genetic or epigenetic events in CRC has identified certain biomarkers that may be utilized to predict behavior and prognosis beyond staging, and inform treatment approaches $(6,7)$. For example, patients with CRC with $B R A F$ V600E mutations often have worse overall survival rates $(8,9)$, and BRAF mutations may predict a lack of response to epidermal growth factor receptor (EGFR) monoclonal antibodies (9), including cetuximab. Furthermore, mitogen activated protein kinase kinase (MEK), is the downstream effector of RAF, and contains two homologous subtypes, MEK1 and MEK2, which are currently the only known activators of extracellular regulated kinase (ERK). Furthermore, MEK protein contains a pocket structure that closes the ATP binding site, making it an ideal target for small molecular inhibitors and a therapeutic strategy in cancer therapy, particularly for those with abnormal activation of the MAPK signaling pathway due to 
$K R A S$ or $B R A F$ mutations. However, a previous study revealed poor results for a MEK inhibitor, trametinib, in patients with $B R A F$-mutated $\mathrm{CRC}$, whereby the overall response rate was $\sim 5 \%$ (10). This result may have been due to the crosstalk activation of receptor tyrosine kinases (RTKs). Indeed, while a single agent targets a single molecular target, cancer cells are often activated via alternate pathways as an escape mechanism to overcome such inhibition, and therefore become resistant to drugs. Lin et al (11) reported that ligand-mediated reactivation of EGFR was involved in the sensitivity reduction in BRAF-mutated non-small cell lung cancer in response to MEK inhibition (11). In addition, Mirzoeva et al (12) reported that MEK inhibitor-treated breast-cancer cells exhibited a strong feedback-activation of EGFR. In summary, release of the MEK-EGFR negative feedback loop may be one of the reasons for decreased sensitivity to MEK inhibitors in cancer, combinatorial drugs that target EGFR and MEK may be a strategy for overcoming MEK inhibitors resistance.

In the present study, a therapeutic strategy to treat a subgroup of CRC cases was investigated. The growth inhibitory effect of a MEK inhibitor, AZD6244, in combination with an EGFR inhibitor, cetuximab, on the regulation of human HT29 CRC cells that harbor the BRAF V600E mutation was assessed in vitro and in an in vivo cell xenograft assay. These findings provide insight regarding preliminary molecular sensitizing mechanisms of cetuximab and AZD6244 for future clinical application in CRC treatment.

\section{Materials and methods}

Cell culture and treatment. The human HT29 CRC cell line $\left(B R A F^{\mathrm{V} 600 \mathrm{E}}\right.$ mutation) was obtained from American Type Culture Collection (Manassas, VA, USA). Cells were maintained in RPMI-1640 (Thermo Fisher Scientific, Inc., Waltham, MA, USA) supplemented with $10 \%$ fetal bovine serum (FBS; Biological Industries, Kibbutz Beit Haemek, Israel), $100 \mu \mathrm{g} / \mathrm{ml}$ penicillin and $100 \mathrm{mg} / \mathrm{ml}$ streptomycin in a humidified incubator with $5 \% \mathrm{CO}_{2}$ at $37^{\circ} \mathrm{C}$.

The selective MEK inhibitor AZD6244 was purchased from Selleck Chemicals (cat. no. S1008; Houston, TX, USA) and dissolved in DMSO at $10 \mathrm{mM}$, then stored at $-20^{\circ} \mathrm{C}$ under light-protected conditions. The EGFR monoclonal antibody cetuximab was supplied by Daping Hospital Cancer Center and Research Institute of Surgery, The Third Military Medical University (Chongqing, China) and used at the indicated doses.

Cell viability Cell Counting kit (CCK)8 assay. HT29 cells in the exponential growth phase were seeded into 96-well plates at a density of $3 \times 10^{3}$ cells/well, incubated for $24 \mathrm{~h}$ and then treated with increasing concentrations of AZD6244 (up to $10^{4} \mathrm{nM}$ ) with/without cetuximab (10 mg/l) for $72 \mathrm{~h}$. Cell viability was assessed by adding $10 \mu \mathrm{l}$ CCK-8 (Bio-Rad Laboratories, Inc., Hercules, CA, USA) into the cell culture and incubating for an additional $2 \mathrm{~h}$. The cell culture medium was then replaced with $200 \mu \mathrm{l} \mathrm{DMSO}$, prior to measurements using a spectrophotometer at $450 \mathrm{nM}$. The cells were treated in triplicate and the experiments were repeated at least three times. The $50 \%$ inhibitory concentration $\left(\mathrm{IC}_{50}\right)$ values were then calculated using the median-effect equation derived by Chou (13).
Flow cytometry analysis for cell cycle and apoptosis. Cells were seeded at a density of $1 \times 10^{5}$ cells $/ 60 \mathrm{~mm}$ well, grown overnight and then treated with vehicle, AZD6244 (120 nM), cetuximab (10 mg/l) or AZD6244 (120 nM) plus cetuximab $(10 \mathrm{mg} / \mathrm{l})$ for $24 \mathrm{~h}$. For cell cycle distribution analysis, cells were washed with PBS twice, fixed in $70 \%$ ice-cold ethanol overnight at $4^{\circ} \mathrm{C}$ and then digested with $100 \mu \mathrm{g} / \mathrm{ml}$ of RNase A at $37^{\circ} \mathrm{C}$ for $30 \mathrm{~min}$. Next, $50 \mu \mathrm{g} / \mathrm{ml}$ of propidium iodide was added to the cell mixture and incubated in the dark for $30 \mathrm{~min}$ at room temperature. For apoptosis analysis, cells were collected and incubated with Annexin V-fluorescein isothiocyanate (FITC) and propidium iodide following the Annexin V-FITC Apoptosis Detection kit protocol (R\&D Systems, Inc., Minneapolis, MN, USA). The fluorescence intensity of each cell sample was measured using the MoFlo ${ }^{\mathrm{TM}}$ XDP flow cytometer (Beckman Coulter, Inc., Brea, CA, USA) and analyzed with KALUZA software v.1.3 (Beckman Coulter, Inc.).

BrdU-labeling assay. HT29 cells in the exponential growth phase were seeded onto coverslips in 6-well plates at a density of $1 \times 10^{5}$ cells/well and grown overnight prior to treatment with vehicle, AZD6244 (120 nM), cetuximab (10 mg/l) or AZD6244 (120 nM) plus cetuximab (10 mg/l) for $24 \mathrm{~h}$. The cells were incubated with RPMI-1640 with FBS $20 \mu \mathrm{M}$ BrdU for $3 \mathrm{~h}$ at $37^{\circ} \mathrm{C}$. At the end of the treatment period, the coverslips were washed with ice-cold PBS and fixed in $70 \%$ ethanol for $10 \mathrm{~min}$ at room temperature. For immunocytochemical staining, the cells were treated with $0.4 \%$ Triton $\mathrm{X}-100$ containing $2 \mathrm{M}$ $\mathrm{HCl}$ in PBS for 45 min and subsequently incubated with the anti-BrdU rat antibody (cat. no. ab6326; Abcam, Cambridge, UK) at a dilution of 1:200 in PBS containing 1\% bovine serum albumin (BSA; GE Healthcare Life Sciences, Little Chalfont, UK; cat. no. CPK1075) overnight in a humidified box at $4^{\circ} \mathrm{C}$. The following day, cells were washed in PBS thrice and further incubated with an FITC-conjugated mouse monoclonal antibody against Rat IgG2a heavy chain (cat. no. 99665; Abcam) at a dilution of 1:400 at room temperature for $1 \mathrm{~h}$. Following washing three times with PBS each $5 \mathrm{~min}$ at room temperature, slides were sealed with $20 \mu 1$ fluorescence sealing solution containing DAPI (Vectashield mounting medium; Vector Laboratories, Inc., Burlingame, CA, USA). Fluorescent images were acquired using a fluorescence microscope at magnification, x100 and FITC-labeled cells were counted in at least five fields of each coverslip.

Western blot analysis. Treated HT29 cells were lysed in RIPA buffer (cat. no. 2010ES60; Beyotime Institute of Biotechnology, Jiangsu, China) on ice at $4^{\circ} \mathrm{C}$ for $10 \mathrm{~min}$ and then centrifuged at $12,000 \mathrm{x} \mathrm{g}$ at $4^{\circ} \mathrm{C}$ to obtain the soluble protein supernatant. Following quantitation with Pierce ${ }^{\mathrm{TM}}$ BCA Protein Assay kit (Thermo Fisher Scientific, Inc.; cat. no. 23227), $50 \mu \mathrm{g}$ protein from each sample were separated by $10 \%$ SDS-PAGE and electrically transferred on to a polyvinylidene difluoride membrane (EMD Millipore, Billerica, MA, USA). For western blot analysis, the membranes were incubated in 5\% BSA at $37^{\circ} \mathrm{C}$ for $1 \mathrm{~h}$ to block potential non-specific binding sites and then incubated overnight at $4^{\circ} \mathrm{C}$ with the primary antibodies against the following: Rabbit monoclonal antibody against phosphorylated (p)-Tyr1068 EGFR (cat. no. 3777; 1:1,000 dilution); rabbit monoclonal antibody against phosphorylated 
(p)-Tyr1289 HER3 (cat. no. 4791; 1:1,000 dilution); rabbit monoclonal antibody against HER3 (cat. no. 12708; 1:1,000 dilution); rabbit monoclonal antibody against p-Ser217/221 MEK (cat. no. 3958; 1:1,000 dilution); rabbit monoclonal antibody against MEK (cat. no. 4694; 1:1,000 dilution); rabbit monoclonal antibody against p-Thr202/204 ERK (cat. no. 4370; 1:2,000 dilution); rabbit polyclonal antibody against ERK (cat. no. 9120; 1:2,000 dilution); rabbit polyclonal antibody against p-Ser473 AKT serine/threonine kinase (AKT; cat. no. 9271; 1:1,000 dilution); rabbit polyclonal antibody against AKT (cat. no. 9272; 1:1,000 dilution); rabbit monoclonal antibody against Cyclin D1 (cat. no. ab134175; 1:10,000 dilution); rabbit polyclonal antibody against PARP (cat. no. ab74290; 1:1,000 dilution); rabbit monoclonal antibody against cleaved-PARP (cat. no. ab320641; 1:1,000 dilution); and mouse monoclonal antibody against $\beta$-actin (cat. no. ab8226; 1:5,000 dilution; all from Cell Signaling Technology, Inc., Danvers, MA, USA). Membranes were subsequently incubated with anti-rabbit (cat. no. 7074; 1:5,000 dilution) or anti-mouse (cat. no. 7076; 1:5,000 dilution) horseradish peroxidase-conjugated secondary antibodies (both from Cell Signaling Technology, Inc.) at room temperature for $1 \mathrm{~h}$. Proteins were detected using Pierce ${ }^{\mathrm{TM}}$ ECL Western Blotting substrate (cat. no. 32106; Thermo Fisher Scientific, Inc.). $\beta$-actin was used as the loading control. Signal intensity with background correction was quantified using Quantity One software v.4.4.0 (Bio-Rad Laboratories, Inc.).

Nude mouse xenograft assay. The animal protocol was approved by the Institutional Animal Care and Use Committee of The Third Military Medical University (Chongqing, China). A total of 24 four-week old female nu/nu nude mice were purchased from Beijing Huafu Kang Biological Technology Co. Inc., (Beijing, China) with an average weight $16.84 \mathrm{~g}$, and maintained in a specific pathogen-free (SPF) 'barrier' facility with controlled at room temperature and 55-62\% humidity, and alternating 12-h light and dark cycles. The mice received SPF mouse chow and were allowed to drink sterile water ad libitum. For the nude mouse xenograft assay, suspended HT-29 cells in sterile PBS were injected into the right flanks of nude mice at $5 \times 10^{6}$ cells per mouse Tumor formation was monitored by caliper measurements twice a week and tumor volume was calculated using the modified ellipsoidal formula: (length/2) x (width) ${ }^{2}$. When xenografts reached an average volume of $200-250 \mathrm{~mm}^{3}$, mice were randomly assigned to the following groups and treated accordingly for 18 days: Vehicle group (once a day, $100 \mu \mathrm{l}$ sterile PBS consisting of $0.2 \%$ Tween-80 and $1 \%$ methycellulose by orogastric gavage; twice a week, $100 \mu \mathrm{l}$ sterile PBS intraperitoneally), cetuximab group (once a day, $100 \mu 1$ sterile PBS consisting of $0.2 \%$ Tween-80 and $1 \%$ methycellulose by orogastric gavage; cetuximab $25 \mathrm{mg} / \mathrm{kg}$ twice a week intraperitoneally), AZD6244 group (once a day, AZD6244 $50 \mathrm{mg} / \mathrm{kg}$ in $0.2 \%$ Tween-80 and $1 \%$ methycellulose in sterile PBS by orogastric gavage; twice a week, $100 \mu$ l sterile PBS intraperitoneally), and a combination of cetuximab plus AZD6244 group (once a day, AZD6244 $50 \mathrm{mg} / \mathrm{kg}$ in $0.2 \%$ Tween-80 and $1 \%$ methycellulose in sterile PBS by orogastric gavage; cetuximab $25 \mathrm{mg} / \mathrm{kg}$ twice a week intraperitoneally). The tumor volumes were monitored every 3 days with a caliper. The tumor volumes repeatedly measured in each mouse were normalized to the initial volume. At the end of experiments, the mice were sacrificed and tumor xenografts were collected for analysis. All animal experiment protocols were approved by the Ethical Committee at the Institute for Experimental Animals of Daping Hospital.

Immunohistochemistry. Tumors were harvested 5 days after treatment as indicated. Tumor xenografts were fixed in a $10 \%$ buffered formalin solution immediately at room temperature for $24 \mathrm{~h}$ following resection from the mice. The tumor blocks were dehydrated and paraffin embedded and then sectioned into $4-\mu \mathrm{m}$ thick slices. For immunohistochemical staining, tissue sections were baked at $60^{\circ} \mathrm{C}$ for $2 \mathrm{~h}$, deparaffinized in xylene, and rehydrated in a descending series of ethanol (100-50\%) and further washed 5 times in $\mathrm{ddH}_{2} \mathrm{O}$ for $1 \mathrm{~min}$ each at room temperature. Slices were then soaked in PBS 2 times at room temperature for $5 \mathrm{~min}$ each. Sections were then boiled in citrate-based antigen unmasking solution at $121^{\circ} \mathrm{C}$ for $1 \mathrm{~min}$ and then cooled down to room temperature, washed with PBS and incubated in a $0.3 \% \mathrm{H}_{2} \mathrm{O}_{2}$ solution in $\mathrm{ddH}_{2} \mathrm{O}$ for $20 \mathrm{~min}$ at room temperature, then washed again in PBS thrice. Next, the sections were incubated in normal goat serum (Wuhan Boster Biological Technology Ltd., Wuhan, China; cat. no. AR0009) for $50 \mathrm{~min}$ at room temperature, followed by incubation with an anti-receptor tyrosine-protein kinase erB-3 (HER3) antibody (cat. no. 12708; Cell Signaling Technology, Inc.) at a dilution of 1:400 with SignalStain Antibody diluent or an anti-p-AKT antibody (cat. no. 4060; Cell Signaling Technology, Inc.) at a dilution of 1:50 at $4^{\circ} \mathrm{C}$ overnight. The following day, the sections were washed with PBS thrice and further incubated with biotin-labeled goat anti-rabbit IgG and HRP-conjugated streptavidin, pre-diluted by the supplier at room temperature (Biotin-Streptavidin HRP Detection Systems; cat. no. SP-9001; Beijing Zhongshan Golden Bridge Biological Technology Co., Ltd., Beijing, China) for $10 \mathrm{~min}$ each. Positive signal was developed in the 3,3-Diaminobenzidine (DAB) substrate solution (0.05\% DAB, $0.015 \% \mathrm{H}_{2} \mathrm{O}_{2}$, PBS) followed by counterstaining at room temperature for $5 \mathrm{~min}$. The stained xenograft sections were reviewed and scored under a light microscope by one of the study investigators. Only evident staining of the cytoplasmic and/or nuclei were reviewed as positive cases. The intensity of staining was classified into four grades: -, no staining; +, definite but weak staining; ++ , moderate staining; and +++ , strong staining.

Statistical analysis. Each experiment was repeated at least three times. All data are presented as the mean \pm standard deviation. Significance was determined using one-way analysis of variance (ANOVA) with Holm-Sidak post-hoc test for multiple comparison correction. $\mathrm{P}<0.05$ was considered to indicate a statistically significant difference. Statistical analyses were performed using SPSS software version 16.0 (SPSS, Inc., Chicago, IL, USA).

\section{Results}

EGFR suppression synergizes MEK inhibitor antitumor activity in HT29 cells. The CCK8 assay data demonstrated that the $\mathrm{IC}_{50}$ of AZD6244 was $108.12 \pm 10.05 \mathrm{nM}$, whereas the $\mathrm{IC}_{50}$ of AZD6244 plus cetuximab was reduced to 
$28.45 \pm 1.922 \mathrm{nM}$, which was approximately a five-fold reduction compared with AZD6244 alone (Fig. 1A). In addition, the BrdU-labeling assay demonstrated that combined treatment with cetuximab and AZD6244 significantly reduced the number of proliferative cells compared with AZD6244 alone $\left(0.6 \pm 0.21 \%\right.$ vs. $2.1 \pm 0.28 \%$; $P=7.87 \times 10^{-5}$; Fig. $1 \mathrm{~B}$ and $\left.\mathrm{C}\right)$. These data indicate that cetuximab enhanced the AZD6244 antitumor activity in HT29 cells in vitro.

Combination of AZD6244 and cetuximab induces cell cycle arrest and apoptosis in HT29 cells. The effects of AZD6244 and cetuximab alone, and in combination on the induction of cell cycle arrest and apoptosis was assessed in HT29 cells. It was revealed that cetuximab alone did not significantly affect the cell cycle distribution in HT29 cells compared with the control, but AZD6244 induced $\mathrm{G}_{0} / \mathrm{G}_{1}$ phase cell cycle arrest, with a significant increase in the G1 phase (73.05 $\pm 4.76 \%$ vs. $88.53 \pm 2.56 \%$; $\mathrm{P}=0.023)$ and decrease in the $\mathrm{S}$ phase $(19.63 \pm 1.28 \%$ vs. $8.73 \pm 2.48 \%$; $\mathrm{P}=0.007)$ (Fig. $2 \mathrm{~A})$. In addition, compared with AZD6244 alone, the combination of AZD6244 and cetuximab significantly increased the number of cells in $\mathrm{G} 1$ phase $(88.53 \pm 2.55 \%$ vs. $93.39 \pm 1.21 \% ; \mathrm{P}=0.080)$ and decreased the number is $\mathrm{S}$ phase $(8.73 \pm 2.48 \%$ vs. $4.24 \pm 0.88 \%$; $\mathrm{P}=0.082$ ) (Fig. 2A). Furthermore, the treatment combination markedly inhibited cyclin D1 expression compared with treatment with AZD6244 alone (Fig. 2B). In addition, it was observed that AZD6244 alone significantly induced apoptosis compared with control (ANOVA $\mathrm{F}=28.72, \mathrm{P}=1.238 \times 10^{-4}$, $8.99 \pm 0.51$ vs. $5.23 \pm 0.66 ; \mathrm{P}=0.004)$. It was also demonstrated that combination of AZD6244 with cetuximab significantly induced tumor cell apoptosis compared with AZD6244 alone $(14.61 \pm 2.67 \%$ vs. $8.99 \pm 0.51 \%$; $\mathrm{P}=0.046$; Fig. 2 C). Consistent with this result, the expression of cleaved poly (ADP-ribose) polymerase (PARP), a hallmark of apoptosis, was also markedly increased in the combination group compared with AZD6244 alone (Fig. 2D). However, cetuximab alone was unable to induce PARP cleavage, while AZD6244 alone slightly induced PARP cleavage in HT29 cells compared with the control.

Combination of AZD6244 and cetuximab abrogates feedback-activation of ERK and AKT. To address the molecular mechanism underlying the synergistic effects of AZD6244 and cetuximab in CRC cells, western blot analysis of MAPK and phosphatidylinositol 3-kinase (PI3K) signaling pathway-associated proteins was performed. It was revealed that p-ERK1/2 levels were transiently inhibited after treatment with AZD6244 and recovered by $24 \mathrm{~h}$, and showed re-accumulation at $48 \mathrm{~h}$ (to $\sim 60 \%$ of the initial level; Fig. 3A). This finding indicates that treatment with AZD6244 leads to reactivation of the MAPK signaling pathway. However, the treatment combination of AZD6244 and cetuximab markedly inhibited p-ERK1/2 and prevented re-activation (Fig. 3B). Although the combination of EGFR inhibitor cetuximab with AZD6244 significantly enhanced the HT29 cell growth inhibition rate, AZD6244 did not increase EGFR phosphorylation (Fig. 3A). However, the total protein expression and phosphorylation of HER3, another member of the HER family, were markedly upregulated following AZD6244 treatment, suggesting that HER3 is activated in response to MEK inhibition (Fig. 3A). In addition, AZD6244 activated p-AKT, an important signaling protein in the PI3K pathway (Fig. 3A). The combination of AZD6244 and cetuximab abrogated the feedback-activation loop of HER3/AKT, which was activated by AZD6244 alone (Fig. 3B).

Combination of AZD6244 and cetuximab increases treatment efficacy in CRC cell xenografts. The effect of the treatment combination was further assessed on nude mice CRC cell xenografts in vivo. The data demonstrated that palpable tumors (200-250 $\mathrm{mm}^{3}$ ) formed in all animals two weeks following the HT29 cell injection. The mice were then treated with vehicle alone, cetuximab (25 mg/kg), AZD6244 (50 mg/kg) or their combination at the same individual dosage $(25$ and $50 \mathrm{mg} / \mathrm{kg}$, respectively) for 18 days. The data indicated that cetuximab alone had no significant effect on the growth of tumor cell xenografts, while AZD6244 alone marginally inhibited tumor growth. In contrast, the treatment combination significantly inhibited growth of HT29 cell xenografts. In addition, treatment with AZD6244 alone or in combination with cetuximab was well-tolerated by mice as no weight loss, or signs of acute or delayed toxicity were observed in the mice (Fig. 4A and B). Furthermore, the tumor volumes at the end of the experiment in the combination treatment group were notably smaller, compared with AZD6244 alone (Fig. 4C). Furthermore, immunohistochemical analysis of xenograft tissues confirmed that the treatment combination suppressed HER3 and p-AKT expression levels compared with AZD6244 alone (Fig. 5). Although the expression of total HER3 was slightly depressed, this result was consistent with the western blotting results as shown in Fig. 3A and B.

\section{Discussion}

The MAPK signaling pathway is a key downstream effector of growth factor receptors and is often dysregulated in a variety of human cancer types. For example, mutations in or overexpression of KRAS and BRAF, together with certain other growth factor receptors, including EGFR and HER2, result in increased cell growth and transformation of malignant cells (14). As MEK is upstream of the ERK and based on the pocket structure near the ATP-binding site in its molecular structure, it is an ideal target for developing small molecule inhibitors (15). In the current study, growth inhibitory effect of the MEK inhibitor AZD6244 was explored in colon cancer HT29 cells. The findings were consistent with data from previous studies $(16,17)$. AZD6244 led to dose-dependent growth inhibition, but the p-ERK levels were only transiently suppressed in HT29 cells in vitro. A previous study revealed that re-activation of the MAPK signaling pathway contributed to the relative insensitivity of the BRAF inhibitor in CRC cells with BRAF mutations (17). The HT29 cells used in the current study also contain a BRAF mutation, and the MEK inhibitor AZD6244 was able to re-activate MAPK signaling. Furthermore, it was observed that AZD6244 treatment alone induced p-AKT levels. Thus, we hypothesize that the poor reactivity of BRAF mutant $\mathrm{CRC}$ cells to the MEK inhibitor AZD6244 is attributed to the re-activation of p-ERK and feedback activation of p-AKT. Although it has been well established that the presence of a BRAF mutation predicts a lack of response to monoclonal EGFR antibodies (9), including 
A

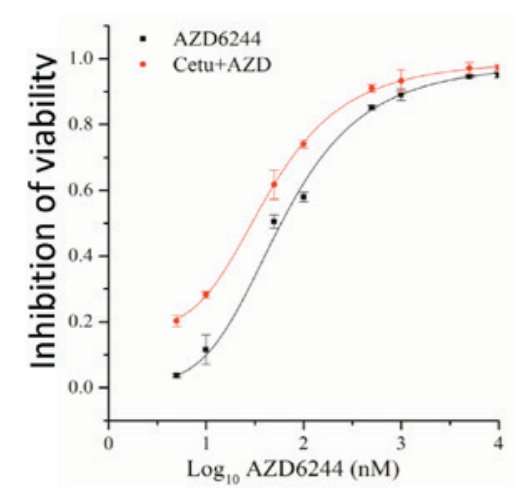

B

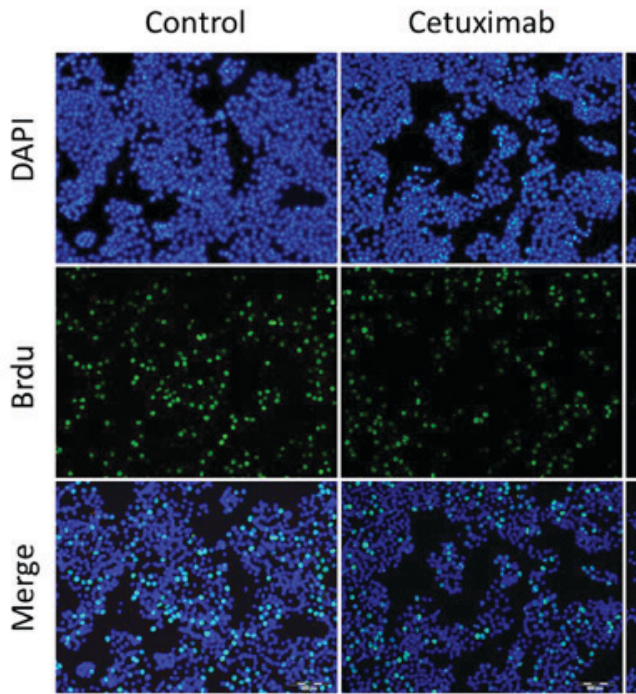

C

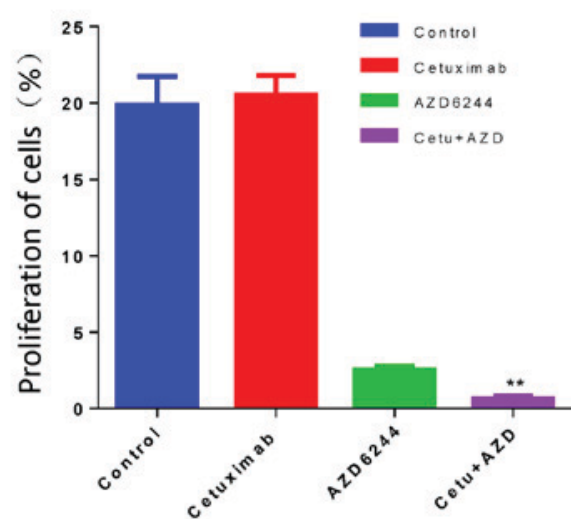

AZD6244

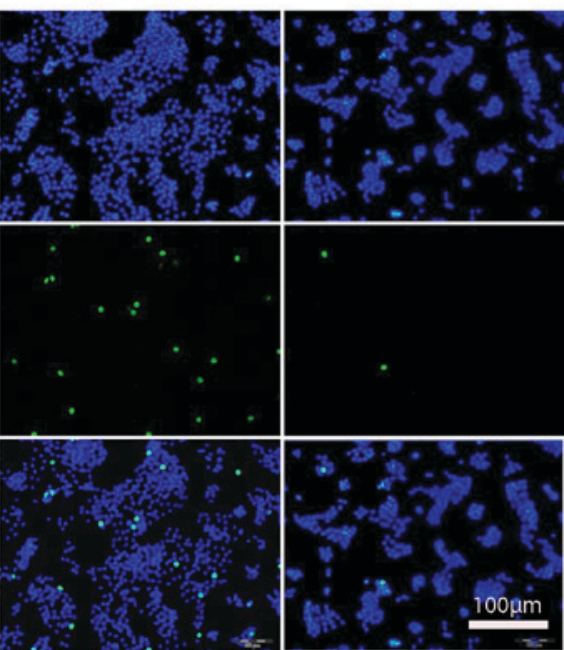

Figure 1. Effects of AZD6244 and cetuximab combination on the inhibition of HT29 cells growth. (A) Cell viability CCK8 assay. HT29 cells were grown and treated with indicated doses of AZD6244 (50-1x10 $\mathrm{nM}$ ) with/without cetuximab (10 mg/l) for $72 \mathrm{~h}$, and subjected to the CCK8 assay. (B) Following the aforementioned treatments, BrdU was added to cells for an additional $2 \mathrm{~h}$ followed by immunostaining with an anti-BrdU antibody. Nuclei were counterstained with DAPI and then quantified as the percentage of proliferative cells (magnification, $\mathrm{x} 100$ ). Data are presented as the mean \pm standard deviation. ${ }^{* *} \mathrm{P}<0.01$ compared with the AZD6244 alone. (C) Representative image of the BrdU assay. CCK8, Cell Counting kit 8; Cetu, cetuximab.

cetuximab, the current data demonstrated that the addition of cetuximab improved the antitumor activity of AZD6244 in HT29 cells that have BRAF mutations. Furthermore, the combination of AZD6244 and cetuximab suppressed p-ERK and p-AKT activation. However, the current data are just proof-of-principle and further studies with more cell lines are required to confirm these results.

Although the combination of cetuximab and AZD6244 significantly enhanced the growth inhibitory effect, and inhibited the re-activation of p-ERK and the feedback activation of $\mathrm{p}-\mathrm{AKT}$, the current study did not observe any significant increase in p-EGFR expression. However, markedly increased levels of total and phosphorylated HER 3 protein expression following AZD6244 treatment in HT29 cells were noted. Functionally, p-HER3 has a high affinity for PI3K and a previous study demonstrated that HER3-mediated activation of the PI3K-AKT pathway led to resistance of non-small cell lung cancer and gastric cancer cells to MEK inhibition $(18,19)$. However, this MAP2K-HER3-AKT negative-feedback loop requires further investigation in other types of cancer. The present study reported the MAP2K-HER3-AKT negative-feedback loop was also presented in colon cancer cells. Furthermore, combinatorial treatment with cetuximab suppressed p-HER3 and p-AKT, indicating that the attenuation of HER3/AKT feedback by cetuximab is partially responsible for the increase of AZD6244 antitumor activity. In addition, a previous structural biology study demonstrated that HER3 is a catalytically impaired member of the HER family and that its signaling is dependent on a hetero-dimerization with a catalytically active partner, in particular, EGFR or HER2 (20). A previous study revealed that EGFR and HER2 signals serve a salvage role, and are associated with resistance to MEK inhibitors in gastric cancer (21). Thus, the current study suggests that the cetuximab-abrogated feedback activation of p-HER3 may be at least partially due to blockage of EGFR/HER3 heterodimer formation. A previous study suggested that the CDC25 downregulation-mediated reactivation of EGFR led to relative insensitivity of BRAF-mutated CRC cells to a BRAF inhibitor (17). In the present study no increase in EGFR activation following AZD6244 treatment was observed; thus, the mechanism of cetuximab-mediated suppression of MAPK re-activation requires further investigation.

The current study also assessed the effects of AZD6244 in combination with cetuximab on the induction of tumor 
A
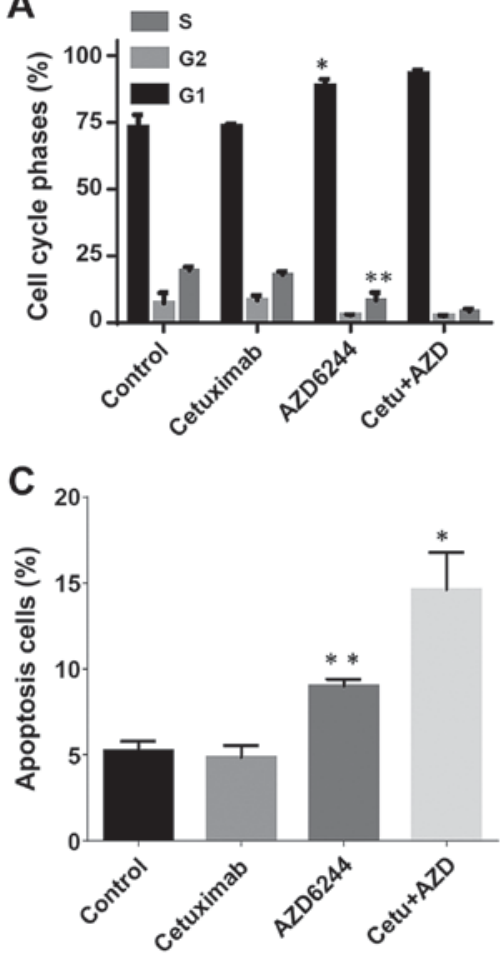

B

HT29

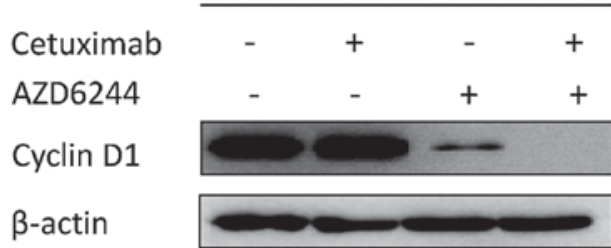

D

Cetuximab

AZD6244

Cleaved-PARP

PARP

$\beta$ - actin
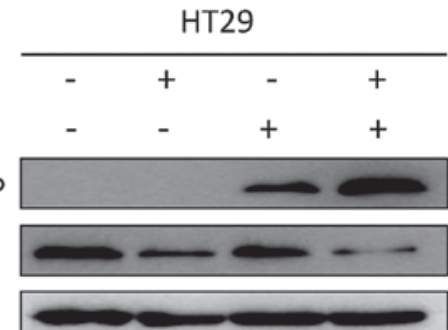

Figure 2. Effects of AZD6244 and cetuximab combination on induction of HT29 cell cycle arrest and apoptosis. HT29 cells were grown and treated with vehicle, cetuximab (10 mg/l), Azd6244 (120 nM) or their combinations for $24 \mathrm{~h}$ and then subjected to (A) flow cytometry of cell cycle distribution, (B) western blot analysis of cycling D1, (C) flow cytometry for apoptosis and (D) western blot analysis of full-length PARP or cleaved-PARP levels. $\beta$-actin was used as a control for western blot analysis Data are presented as the mean \pm standard deviation. (A) ${ }^{*} \mathrm{P}<0.05,{ }^{{ }^{* *}} \mathrm{P}<0.01$ compared with the control group. (B) ${ }^{*} \mathrm{P}<0.05$ compared with the AZD6244 alone; ${ }^{* *} \mathrm{P}<0.01$ compared with the control group. Cetu, cetuximab; PARP, poly (ADP-ribose) polymerase.

A

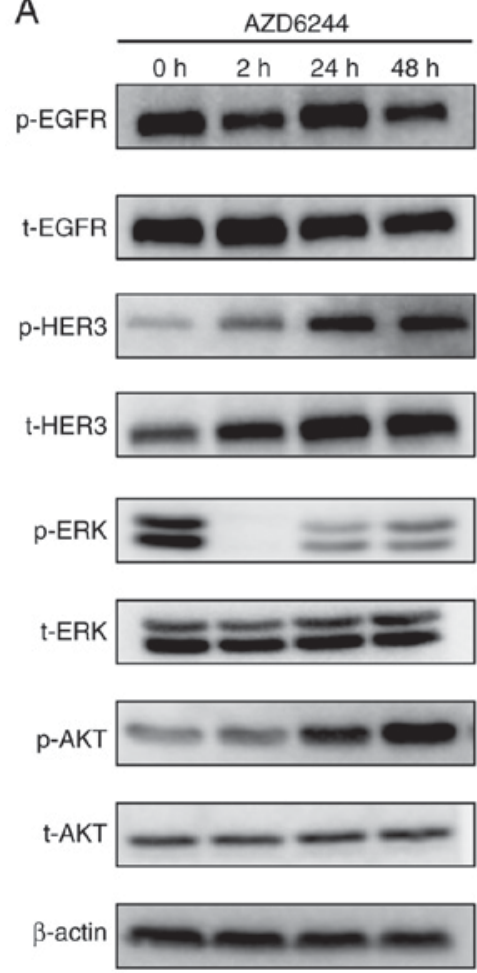

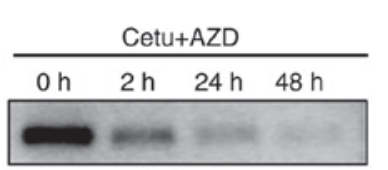
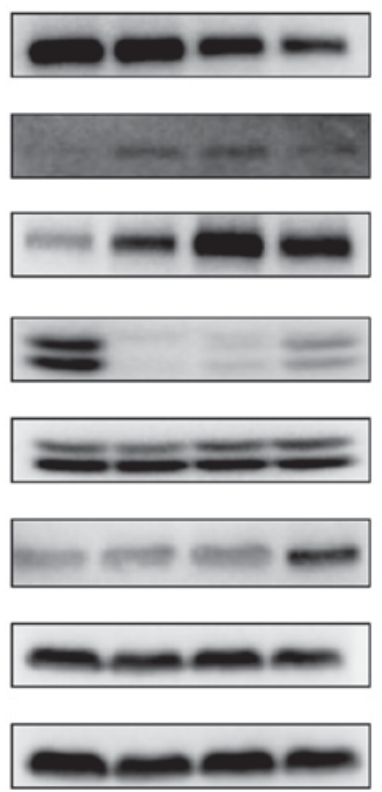

B

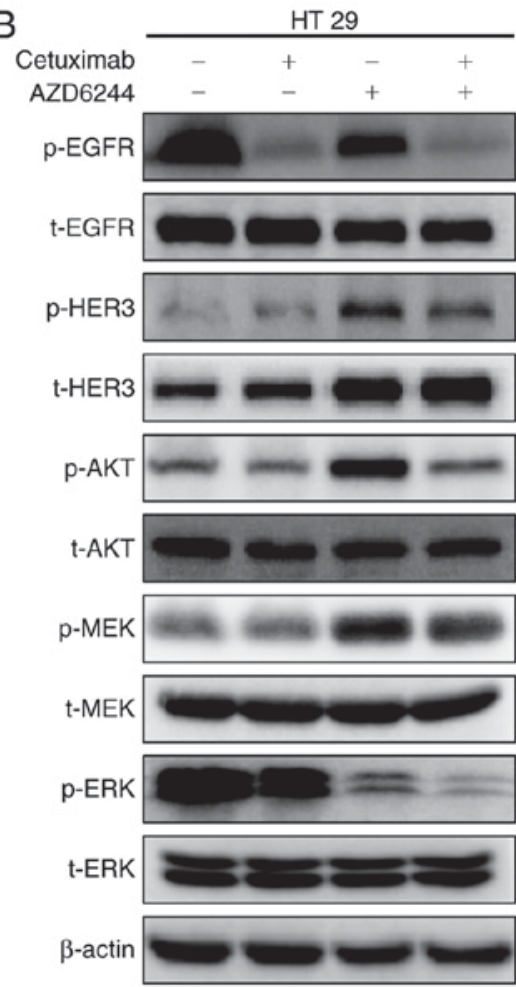

Figure 3. Effects of AZD6244 and cetuximab combination on suppression of re-activated p-ERK and feedback activated p-AKT. (A) HT29 cells were treated with AZD6244 $(120 \mathrm{nM})$ with or without cetuximab $(10 \mathrm{mg})$ at the indicated time points, after which whole-cell extracts were blotted with antibodies against p-EGFR, total-EGFR, p-HER3, total-HER3, p-MEK1/2, total-MEK1/2, p-ERK, total-ERK, p-AKT and total-AKT. (B) HT29 cells were treated with vehicle, cetuximab (10 mg), AZD6244 (120 nM) or cetuximab $(10 \mathrm{mg})$ plus AZD6244 $(120 \mathrm{nM})$ for $24 \mathrm{~h}$, after which whole-cell extracts were blotted with p-EGFR, total-EGFR, p-HER3, total-HER3, p-MEK1/2, total-MEK1/2, p-ERK, total-ERK, p-AKT and total-AKT. Cetu, cetuximab; p, phosphorylated; t, total; AKT, AKT serine/threonine kinase; EGFR, epidermal growth factor receptor; HER3, receptor tyrosine-protein kinase erB-3; MEK, mitogen-activated protein kinase kinase; ERK, extracellular regulated kinase. 
A

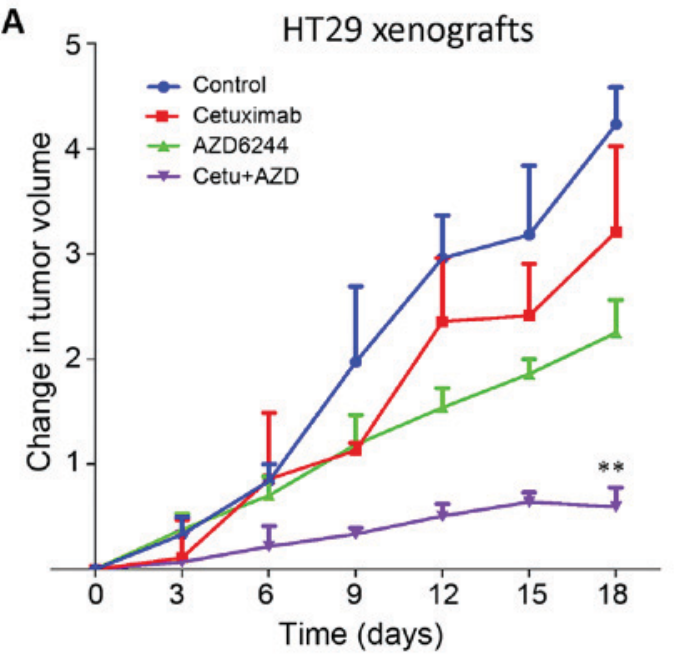

C
B

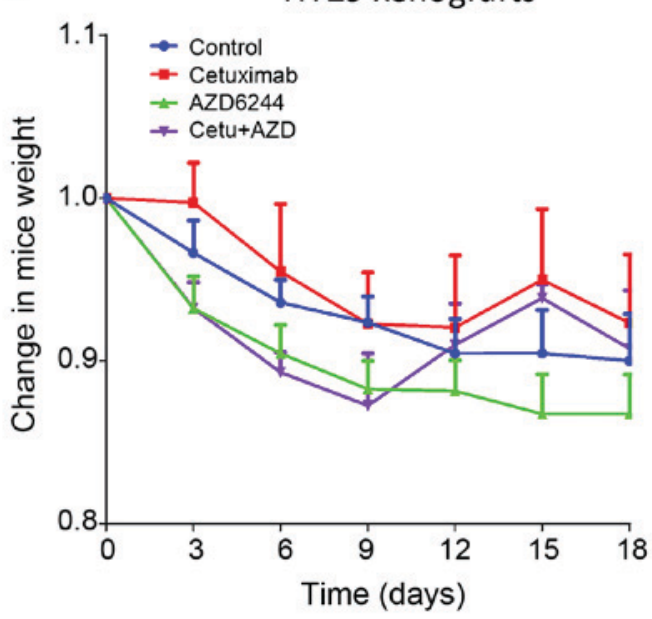

AZD6244

HT29 xenografts

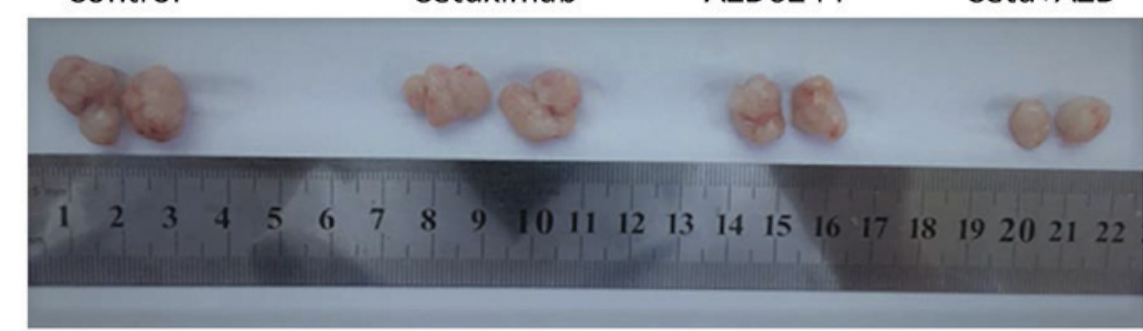

Figure 4. Effects of AZD6244 and cetuximab combination on suppression of colorectal cancer HT29 cell xenograft formation in nude mice. (A) HT29 cell xenografts were grown to $200-250 \mathrm{~mm}^{3}$ in size and then the mice were treated with vehicle, cetuximab (25 mg/ $\left.\left.\mathrm{kg}\right), \mathrm{AZD} 6244(50 \mathrm{mg} / \mathrm{kg}) \mathrm{or} \mathrm{cetuximab} \mathrm{(25} \mathrm{mg/} \mathrm{kg}\right)$ plus AZD6244 $(50 \mathrm{mg} / \mathrm{kg})$ for 18 days, and tumor xenografts volumes were plotted. The tumor volumes repeatedly measured in each mouse were normalized to the initial volume. Data are presented as the mean relative fold changes \pm standard error. ${ }^{* *} \mathrm{P}<0.01$ compared with AZD6244 alone. (B) Following treatment with the drugs, mouse weight was measured twice a week using an electronic balance. Data are presented as the mean mouse weights \pm standard error. (C) Xenografts after 18 days of treatment. Selected xenograft gross images of representative cases are presented after sacrifice of the mice. Cetu, cetuximab.

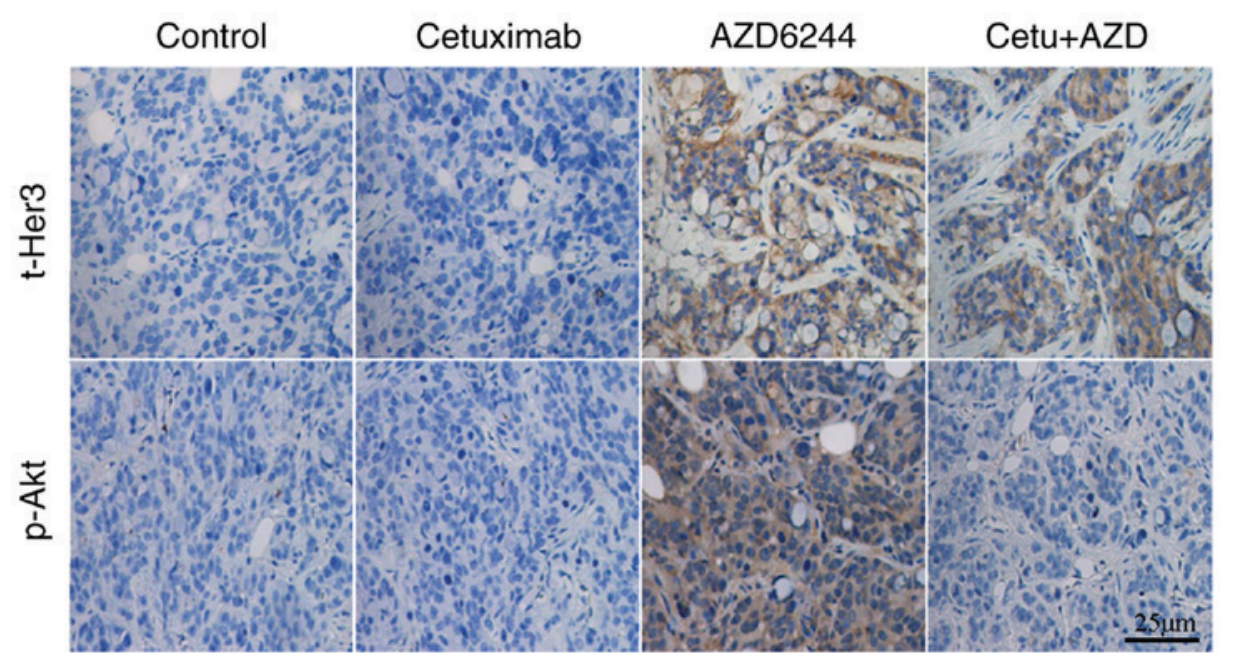

Figure 5. Effects of AZD6244 and cetuximab combination on regulation of p-AKT and t-HER3 expression in xenografts. HT29 cell xenografts were resected from the mice after 5 days of treatment with indicated agents and processed for immunohistochemical analysis of p-AKT and t-HER3 expression (magnification, x200). Cetu, cetuximab; p, phosphorylated; t, total; AKT, AKT serine/threonine kinase; HER3, receptor tyrosine-protein kinase erB-3.

cell cycle arrest and apoptosis. It was demonstrated that the combinatorial treatment further increased the number of tumor cells arrested at G1 phase and decreased the cells in $\mathrm{S}$ phase, accompanied by a significantly increased percentage of apoptotic cells. These data were supported by inhibition of cyclin D1 expression and induction of cleaved PARP in HT29 cells following combinatorial treatment. In addition, the nude mouse xenograft assay further confirmed the in vitro data, indicating that the combination of AZD6244 and cetuximab may have merit as a treatment strategy for human patients with CRC. However, further studies are required to confirm whether 
the combinatorial treatment only works in BRAF-mutated CRC cells.

Due to the crosstalk between the RTK-signaling pathways, no signal gene dependency is to be expected. Numerous studies have reported that when cancer cells are treated with drugs that block single molecular target, they are often able to relief of feedback and activate alternate pathways as escape mechanisms to overcome the blockade. Combined inhibition of specific pathways induced by negative feedback should be an effective strategy to enhance antitumor activity (22-24). Chandarlapaty et al (22) reported that AKT inhibitor relieves the feedback suppression of RTK, combined inhibition of AKT and HER kinase activity may improve anticancer activity. In this current study, it was determined that the feedback activity of HER3/AKT contributes to the HT29 cells relative insensitivity to MEK inhibitor AZD6244, combination of AZD6244 and cetuximab can abrogate the HER3/AKT feedback activation and improve the antitumor activity of AZD6244. These findings suggest that co-inhibition of MEK and EGFR may be a promising treatment strategy in CRCs, at least those that possess a BRAF mutation. Although this work suggests that HER3-mediated feedback re-activation of p-AKT partially contributes to HT29 cell insensitivity to AZD6244, we hypothesize that additional growth factors may contribute to this treatment resistance. In our future studies, the mechanism of combinatorial treatment will be further explored.

\section{Acknowledgements}

Not applicable.

\section{Funding}

The present study was supported in part by a grant from the foundation of the Chinese Society of Clinical Oncology (grant no. Y-MX2015-041).

\section{Availability of data and materials}

The datasets used and/or analyzed during the current study are available from the corresponding author on reasonable request.

\section{Authors' contributions}

QZ and HX made substantial contributions to conception and design of the present study. FJ, ML and JL were all responsible for acquisition of data, or analysis and interpretation of data. GW was involved in drafting the manuscript,revising it critically for important intellectual content and helped to design the study. All authors made the final approval of the version to be published.

\section{Ethics approval and consent to participate}

Animal experiment protocols were approved by the ethical committee at the Institute for Experimental Animals of Daping Hospital.

\section{Consent for publication}

Not applicable.

\section{Competing interests}

The authors declare that they have no competing interests.

\section{References}

1. Torre LA, Bray F, Siegel RL, Ferlay J, Lortet-Tieulent J and Jemal A: Global cancer statistics, 2012. CA Cancer J Clin 65: 87-108, 2015.

2. Arnold M, Sierra MS, Laversanne M, Soerjomataram I, Jemal A and Bray F: Global patterns and trends in colorectal cancer incidence and mortality. GUT 66: 683-691, 2017.

3. US Preventive Services Task Force; Bibbins-Domingo K, Grossman DC, Curry SJ, Davidson KW, Epling JW Jr, García FAR, Gillman MW, Harper DM, Kemper AR, et al: Screening for colorectal cancer: US preventive services task force recommendation statement. JAMA 315: 2564-2575, 2016.

4. Siegel RL, Miller KD, Fedewa SA, Ahnen DJ, Meester RGS, Barzi A and Jemal A: Colorectal cancer statistics, 2017. CA Cancer J Clin 67: 177, 2017.

5. McGuire S: World cancer report 2014. Geneva, Switzerland: World Health Organization, International agency for research on cancer, WHO Press, 2015. Adv Nutr 7: 418-419, 2016.

6. Guinney J, Dienstmann R, Wang X, de Reyniès A, Schlicker A, Soneson C, Marisa L, Roepman P, Nyamundanda G, Angelino P, et al: The consensus molecular subtypes of colorectal cancer. Nat Med 21: 1350-1356, 2015.

7. Carethers JM and Jung BH: Genetics and genetic biomarkers in sporadic colorectal cancer. Gastroenterology 149: 1177-1190.e3, 2015.

8. Richman SD, Seymour MT, Chambers P, Elliott F, Daly CL, Meade AM, Taylor G, Barrett JH and Quirke P: KRAS and BRAF mutations in advanced colorectal cancer are associated with poor prognosis but do not preclude benefit from oxaliplatin or irinotecan: Results from the MRC FOCUS trial. J Clin Oncol 27: 5931-5937, 2009.

9. Di Nicolantonio F, Martini M, Molinari F, Sartore-Bianchi A, Arena S, Saletti P, De Dosso S, Mazzucchelli L, Frattini M, Siena S and Bardelli A: Wild-type BRAF is required for response to panitumumab or cetuximab in metastatic colorectal cancer. J Clin Oncol 26: 5705-5712, 2008.

10. Infante JR,Fecher LA, Falchook GS, Nallapareddy S, Gordon MS, Becerra C, DeMarini DJ, Cox DS, Xu Y, Morris SR, et al: Safety, pharmacokinetic, pharmacodynamic, and efficacy data for the oral MEK inhibitor trametinib: A phase 1 dose-escalation trial. Lancet Oncol 13: 773-781, 2012.

11. Lin L, Asthana S, Chan E, Bandyopadhyay S, Martins MM, Olivas V, Yan JJ, Pham L, Wang MM, Bollag G, et al: Mapping the molecular determinants of BRAF oncogene dependence in human lung cancer. Proc Natl Acad Sci USA 111: E748-E757, 2014.

12. Mirzoeva OK, Das D, Heiser LM, Bhattacharya S, Siwak D, Gendelman R, Bayani N, Wang NJ, Neve RM, Guan Y, et al: Basal subtype and MAPK/ERK kinase (MEK)-phosphoinositide 3-kinase feedback signaling determine susceptibility of breast cancer cells to MEK inhibition. Cancer Res 69: 565-572, 2009.

13. Chou TC: Drug combination studies and their synergy quantification using the Chou-Talalay method. Cancer Res 70: 440-446, 2010.

14. Rubinfeld $\mathrm{H}$ and Seger R: The ERK cascade: A prototype of MAPK signaling. Mol Biotechnol 31: 151-174, 2005.

15. Misale S, Arena S, Lamba S, Siravegna G, Lallo A, Hobor S, Russo M, Buscarino M, Lazzari L, Sartore-Bianchi A, et al: Blockade of EGFR and MEK intercepts heterogeneous mechanisms of acquired resistance to anti-EGFR therapies in colorectal cancer. Sci Transl Med 6: 224ra26, 2014.

16. Friday BB, Yu C, Dy GK, Smith PD, Wang L, Thibodeau SN and Adjei AA: BRAF V600E disrupts AZD6244-induced abrogation of negative feedback pathways between extracellular signal-regulated kinase and Raf proteins. Cancer Res 68: 6145-6153, 2008.

17. Prahallad A, Sun C, Huang S, Di Nicolantonio F, Salazar R, Zecchin D, Beijersbergen RL, Bardelli A and Bernards R: Unresponsiveness of colon cancer to BRAF(V600E) inhibition through feedback activation of EGFR. Nature 483: 100-103, 2012.

18. Yoon YK, Kim HP, Han SW, Hur HS, Oh DY, Im SA, Bang YJ and Kim TY: Combination of EGFR and MEK1/2 inhibitor shows synergistic effects by suppressing EGFR/HER3-dependent AKT activation in human gastric cancer cells. Mol Cancer Ther 8: 2526-2536, 2009. 
19. Okimoto RA, Lin L, Olivas V, Chan E, Markegard E, Rymar A, Neel D, Chen X, Hemmati G, Bollag G and Bivona TG: Preclinical efficacy of a RAF inhibitor that evades paradoxical MAPK pathway activation in protein kinase BRAF-mutant lung cancer. Proc Natl Acad Sci USA 113: 13456-13461, 2016.

20. Littlefield P, Liu L, Mysore V, Shan Y, Shaw DE and Jura N: Structural analysis of the EGFR/HER3 heterodimer reveals the molecular basis for activating HER3 mutations. Sci Signal 7: a114, 2014.

21. Mizukami T, Togashi Y, Sogabe S, Banno E, Terashima M, De Velasco MA, Sakai K, Fujita Y, Tomida S, Nakajima TE, et al: EGFR and HER2 signals play a salvage role in MEK1-mutated gastric cancer after MEK inhibition. Int J Oncol 47: 499-505, 2015.

22. Chandarlapaty S, Sawai A, Scaltriti M, Rodrik-Outmezguine V, Grbovic-Huezo O, Serra V, Majumder PK, Baselga J and Rosen N: AKT inhibition relieves feedback suppression of receptor tyrosine kinase expression and activity. Cancer Cell 19: 58-71, 2011.
23. E J, Xing J, Gong H, He J and Zhang W: Combine MEK inhibition with PI3K/mTOR inhibition exert inhibitory tumor growth effect on KRAS and PIK3CA mutation CRC xenografts due to reduced expression of VEGF and matrix metallopeptidase-9. Tumour Biol 36: 1091-1097, 2015.

24. Haagensen EJ, Kyle S, Beale GS, Maxwell RJ and Newell DR: The synergistic interaction of MEK and PI3K inhibitors is modulated by mTOR inhibition. Br J Cancer 106: 1386-1394, 2012.

(i) () () This work is licensed under a Creative Commons CY No No Attribution-NonCommercial-NoDerivatives 4.0 International (CC BY-NC-ND 4.0) License. 